\title{
A Survey Paper on Iris Based Access System Using Stabilized Iris Encoding and Zernike Moments Phase Features
}

\author{
Ms. Payal Mahajan ${ }^{1}$, Mrs. Nandini Dhole ${ }^{2}$ \\ Student (ME), Electronics and Telecommunication, R.M.D Sinhgad School of Engineering, Pune, India ${ }^{1}$ \\ Asst. Professor, Electronics and Telecommunication, R.M.D Sinhgad School of Engineering, Pune, India ${ }^{2}$
}

\begin{abstract}
Iris recognition is a technique for biometric identification. Biometric identification gives programmed acknowledgment of an individual based on the unique feature of physiological characteristics or behavioral characteristic. Iris acknowledgment is a technique for perceiving a man by examining the iris design. This review paper covers the distinctive iris acknowledgment systems and strategies. The standardized area is proposed, which essentially contains the Zernike moment (ZM) phase An exact and vigorous estimation of the rotation angle between a pair of normalized regions is then described and used to measure the comparability between two coordinating areas. Exact iris acknowledgment from the indirectly gained face or eye pictures requires improvement of powerful systems, which can represent for significant variations in the segmented iris image quality. Such variations can be highly correlated with the consistency of encoded iris elements and information that such delicate bits can be misused to enhance coordinating exactness.
\end{abstract}

Keywords: Biometrics, iris recognition, less-constrained iris recognition, at-a-distance iris recognition.

\section{INTRODUCTION}

Automated iris acknowledgment for remotely gained images utilizing visible imaging under less obliged conditions has gotten extraordinary consideration as of late. Reception of the unmistakable imaging in the acquisition stage gives a couple points of interest over the traditional NIR-based iris acknowledgment frameworks [1]. Biometrics is commonly characterized as the investigation of strategies for making estimations of physical, organic or behavioral traits that can be utilized to recognize a man. Inside the field of biometrics, fingerprint, face and Iris are often thought of as the current significant universally useful techniques [2].

IRIS recognition has developed as one of the most promising technologies to give reliable human identification. Remarkable iris recognition precision has been accounted for by the current best in class iris acknowledgment algorithms on the iris images obtained utilizing close infrared (NIR) imaging from controlled condition. The pictures gained utilizing obvious imaging under less controlled situations are frequently noisier as compared to those acquired under NIR illumination and therefore require improvement of iris coordinating procedures which are more tolerant to noise. Iris recognition from indirectly obtained eye images and under less constrained environments represents a few difficulties, particularly for the images obtained utilizing Unmistakable imaging. Picture quality degradation isusually severer in the visible illumination eye images acquired from such dynamic environments.

\section{LITERATURE SURVEY}

C.-W. Tan, et al. [1] "Towards online iris and periocular recognition under relaxed imaging constraints," This paper has exhibited a promising methodology for computerized human acknowledgment by at the simultaneously exploiting iris and periocular features to give enhanced acknowledgment execution. The created iris segmentation approach is computationally attractive than already proposed approaches for obvious brightening databases. However, further efforts are as yet required to enhance the proficiency of the iris segmentation algorithm with a specific end goal to make it feasible for any possible online application/deployment (e.g. remote surveillance).

The exploratory outcomes acquired from the three openly accessible at-a-separate databases, i.e., UBIRIS.v2, FRGC and CASIA.v4-distance, clearly demonstrate the superior performance of the proposed segmentation system, which recommend the normal change of $9.5 \%, 4.3 \%$ and $25.7 \%$ in segmentation precision individually for the three utilized databases. With a specific end goal to enhance the acknowledgment exactness, this paper abuses the joint technique utilizing the iris and periocular features. Rigorous experiments were carried out to evaluate the performance of different periocular feature extraction techniques on the three utilized databases. The best learned joint features from the experiments are also compared with the existing state-of-art algorithms, evaluated utilizing the three utilized databases, which revealed a general change of $52.4 \%$ in rank-one acknowledgment exactness. 
K. W. Bowyer, et al. [2] "The results of the NICE. II iris biometrics competition,"Biometrics is regularly characterized as the investigation of strategies for making estimations of physical, natural or behavioral traits that can be utilized to distinguish a man. Inside the field of biometrics, fingerprint, face and Iris are often thought of as the current major general-purpose strategies. The second period of the Noisy Iris Challenge Evaluation attracted participation by 67 research groups from around the world. In contrast to all current commercial Iris biometrics innovation, the NICE competitions focus on performing Iris biometrics on visible-light images. While NICE.I focused on segmentation, NICE.II focused on performance in feature extraction and matching. The eight top-performing algorithms from NICE.II are considered, and proposals are made for lessons that can be drawn from the outcomes.

S. Venugopalanet al [3] "Long range iris acquisition system for stationary mobile and mobile stationary subjects," Most iris based biometric frameworks require a considerable measure of collaboration from the clients with the goal that iris images of acceptable quality might be obtained. Features from these may then be utilized for acknowledgment purposes. Generally less works in literature address the question of less cooperative iris acquisition frameworks with a specific end goal to lessen imperatives on clients. In this paper, we describe our ongoing work in designing and developing such a framework. In this paper, we've illustrated the plan and improvement of a framework to procure high determination face and irisimagesfrom mobile (or stationary) subjects using a single imaging sensor. For portable subjects, we have incorporated an optional sensor fitted with a wide angle lens to track his/her movements during the obtaining procedure. As the subject moves towards the framework, a kalman filter based face tracking componentmoves a pan/tilt mechanism.

Y. Zhou, et al. [4] "Personal identification from iris images using localized radon transform," This paper has explored another approach for the individual identification utilizing confined Radon change (LRT). The introduction subtle elements of the exceptional iris surfaces were removed utilizing LRT. The test comes about exhibited in this paper utilizing LRT are exceedingly encouraging on both of the two open databases, i.e. accomplish an EER of $0.53 \%$ and $2.82 \%$ on IITD v1 and CASIA v3 databases separately (CASIA v1 database was additionally examined, and the subsequent EER was $0.24 \%$, result are not introduced to monitor space). The proposed approach requires fundamentally littler computational operation for feature extraction and therefore highly suitable for applications where the speed is of prime consideration. Our investigation proposes that LRT based feature extraction approach requires about $2 \mathrm{Kw}$ times $(\mathrm{K}$ and $\mathrm{w}$ stands for the filter size and chosen line width) less operations when contrasted with those from the routine Gabor filter based approach.
H. Proenca, et al. [5] "Iris recognition: On the segmentation of shattered images acquired in the visible wavelength," iris recognition imaging constraints are receiving increasing attention. There are several recommendations to create frameworks that work in the obvious wavelength and in less obliged situations. These imaging conditions cause obtained noisy artifacts that lead to severely degraded images, making iris segmentation a major issue. Due to favorable comparisons with other biometric traits, the popularity of the iris has developed extensively and endeavors are gathered in the advancement of frameworks that are less obliged to subjects, utilizing images captured at-a-distance and onthe-move. These are extremely ambitious conditions that lead to severely degraded image data, which can be particularly trying for image segmentation. Our method encompasses three tasks that are normally isolated in the literature: eye detection, discrimination of the noise-free iris texture iris segmentation, and iris segmentation. Our key insight is 1) to consider the sclera as the most easily distinguishable part of the eye in the case of degraded images and 2) to exploit the mandatory adjacency between the sclera and the iris to propose a new type of feature (proportion of sclera) that is key in the restriction of the iris, through a machine learning characterization approach. Z. He, et al. [6] "Toward accurate and fast iris segmentation for iris biometrics," Iris segmentation is an basic module in iris acknowledgment since it characterizes the viable image region utilized for resulting preparing,for example, feature extraction. Traditional iris segmentation techniques regularly include a comprehensive search of a large parameter space, which is time consuming and sensitive to noise. To address these issues, this paper displays a novel algorithm for exact and quick iris segmentation. In this paper, we have exhibited a precise and quick iris segmentation algorithm for iris biometrics. There are five major contributions. To start with, we build up a novel reflection removal strategy to reject specularities in the information pictures and an Adaboostcascade iris detector to detect the iris in them. Four envelop points are calculated to bilinearly interpolate specular reflections, paving the way for efficient iris identification. With the educated Adaboostcascade iris identifier, non-iris images are excluded before further processing so that unnecessary computation is avoided. In addition, a rough iris center is extracted in iris images, which gives critical signals to ensuing preparing.

K. W. Bowyer, et al. [7] "Image understanding for iris biometrics: A survey,"At whatever point individuals sign onto PCs, get to an ATM, go through air terminal security, utilize credit cards, or enter high-security areas, they need to confirm their characters. Individuals commonly utilize client names, passwords, and ID cards to demonstrate that they are who they claim to be. However, passwords can be forgotten, and identification cards can be lost or stolen. This review covers the historical improvement and current state of the art in image understanding for iris biometrics. Most research productions can be arranged as making their 
essential contribution to one of the four major modules in iris biometrics: image acquisition, iris segmentation, texture analysis and matching of texture representations. Other essential research incorporates test assessments, image databases, applications and frameworks, and therapeutic conditions that may influence the iris. We likewise propose a short list of recommended readings for someone new to the field to quickly grasp the big picture of iris biometrics.

J. Daugman, et al. [8] "New methods in iris recognition,"This paper presents the following four advances in iris acknowledgment: 1) more trained strategies for identifying and reliably demonstrating the iris inward and external limits with dynamic forms, prompting more adaptable implanted arrange frameworks; 2) Fourier-based techniques for tackling issues in iris trigonometry and projective geometry, allowing off-axis gaze to be handled by detecting it and "rotating" the eye into orthographic perspective; 3) statistical inference techniques for distinguishing and barring eyelashes; and 4) investigation of score normalizations, depending on the measure of iris information that is accessible in images and the required scale of database search. Measurable outcomes are displayed in view of 200 billion iris crosscorrelations that were produced from 632500 irises in the United Arab Emirates database to investigate the standardization issues brought up in various areas of receiver operating characteristic curves.

J. R. Matey et al. [9] "Iris on the move: Acquisition of images for iris recognition in less constrained environments," Iris recognition is one of the most effective methods for biometric identification ever developed. Commercial frameworks in view of the algorithms developed by John Daugman have been available since 1995 and have beenutilizedinan variety of practical applications. In any case, all as of now accessible frameworks impose substantial constraints on subject position and motion during the recognition procedure. These constraints are largely driven by the image acquisition process, rather than the specific example coordinating algorithm utilized for the acknowledgment procedure. In this paper we show consequences of our endeavours to significantly reduce constraints on position and motion by means of a new image acquisition framework in view of high-determination cameras, video synchronized strobed brightening, also, specularity based image segmentation. The IOM framework is the to begin with, and as of now as it were, framework that can capture iris images of recognition quality from subjects walking at a normal pace through a minimally confining portal. This outcome has been accomplished using controlled, strobed enlightenment, high-resolution cameras, and specularity based calculations for eye finding.

J. Daugman, et al. [10] "How iris recognition works," Algorithms created by the creator for perceiving people by their iris designs have now been tried in numerous field and research center trials, delivering no false matches in a few million examination tests. The recognition principle is the failure of a test of statistical independence on iris phase structure encoded by multi-scale quadrature wavelets. The combinatorial complexity of this phase data crosswise over various people traverses about 249 degrees of opportunity and produces a discrimination entropy of about $3.2 \mathrm{~b} \mathrm{~mm} 2$ over the iris, enabling real-time decisions about individual personality with to a great degree high certainty. The high certainty levels are imperative since they permit huge databases to be looked thoroughly (oneto-many "identification mode") without making false matches, despite so many chances. Biometrics that lacks this property can only survive one-to-one ("verification") or few comparisons. This paper clarifies the Iris recognition algorithms and presents results of 9.1 million comparisons among eye images from trials in Britain, the USA, Japan, and Korea.

\section{PROPOSED SYSTEM}

In today's world security is becoming more and more important. Confirmation assumes a noteworthy part in security. Verification is the way toward confirming the guaranteed character of a man. Validation is a methods for barrier against intruders. There are of different sorts like verification utilizing username with secret key, utilizing card and utilizing biometric. Most normally, username with password is utilized for verification, yet Password is effortlessly carked or stolen in view of human inclination to make secret word simple to recall and furthermore note down the watchword so that there is no compelling reason to recollect. Cards can be stolen and accessing by anyone. Therefore there is no way of knowing that the claimed person is the one. Biometric identification provides secure authentication of a person as data can't be steal and duplicated. Biometric information is exceptional and forever connected with a man. Iris recognition is a strategy for biometric identification gives automatic recognition of an individual based on the unique feature of physiological characteristics like fingerprints, palm, DNA, face, iris, retina and vein or behavioral characteristic like Handwriting, speech and signature.

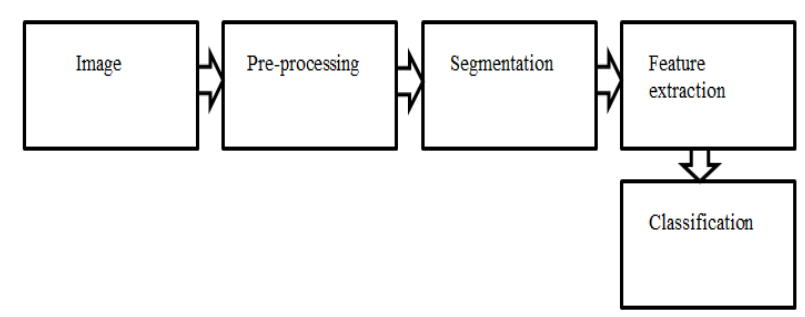

Fig 1: block diagram of proposed system

Iris recognition is a strategy of recognizing a person by analyzing the iris pattern. Iris example is framed by six months after birth. Iris design stays stable following a year and continues as before forever time that implies it doesn't have maturing impact. Iris examples of identical twins 
differ and a person's left and right eyes have different patterns as well. This separate it from palm print or fingerprints or palm, which can be difficult to recognize after years of certain types of manual labor. It is regarded as the most reliable biometric technology since iris is highly distinctive and robust. Effective and powerful iris Segmentation algorithm is crucial for any successful iris recognition procedure with a specific end goal to naturally remove iris area from the eye image. Fig. demonstrates the significant iris division steps utilized in this paper to consequently limit the iris area. The iris segmentation process starts with the image enhancement using retinex algorithm. Retinex algorithm is order to improve the contrast between limbic and pupillary boundaries.

\section{IV.CONCLUSION}

This paper has researched a promising iris encoding and coordinating procedure for the iris images acquired at-adistance, utilizing both NIR and visible imaging, under less constrained environments. Such the images gained ata-separate and under less constrained imaging conditions are regularly degraded because of noise presented by various sources, and in this manner it will probably distort 25 the iris texture details (e.g. scale, rotation, blur, offangle, occlusion, etc.). In this way, the segmented iris images following the iris normalization step reveals the distorted texture details which can be varying even for the iris images from the same class. The joint methodology displayed in this paper abuses a worldwide iris bits adjustment encoding system and a limited ZMs stage based encoding technique to heartily recoup the iris features. Our methodology has been to at the same time discover the coordinating data from local region pixels (which is more tolerant to the distortion) while likewise assessing the coordinating data for the elements which can protect worldwide matches from more steady surface examples/locales. The worldwide iris encoding is to a great extent in view of the current promising exertion on the delicate bits estimation. The strength of the encoded iris elements can be much corresponded with the consistency of the subsequent iris bits, which can be assessed from the information of the delicate bits. A nonlinear approach which can all the more viably record for both consistencies of the iris bit and also for the overall quality of the weight map is employed to stabilize/weight the encoded iris bits.

\section{REFERENCES}

[1] C.-W. Tan and A. Kumar, "Towards online iris and periocular recognition under relaxed imaging constraints," IEEE Trans. Image Process., vol. 22,no. 10, pp. 3751-3765, Oct. 2013.

[2] K. W. Bowyer, "The results of the NICE. II iris biometrics competition," Pattern Recognit. Lett., vol. 33, no. 8, pp. 965-969, 2012.

[3] S. Venugopalanet al., "Long range iris acquisition system for stationaryand mobile subjects," in Proc. IEEE Int. Joint Conf. Biometrics Compendium(IJCB), Oct. 2011, pp. 1-8.

[4] Y. Zhou and A. Kumar, "Personal identification from iris images usinglocalized radon transform," in Proc. 20th Int. Conf. Pattern Recognit.(ICPR), Aug. 2010, pp. 2840-2843.
[5] H. Proenca, "Iris recognition: On the segmentation of degraded imagesacquired in the visible wavelength," IEEE Trans. Pattern Anal. Mach.Intell., vol. 32, no. 8, pp. 1502-1516, Aug. 2010.

[6] Z. He, T. Tan, Z. Sun, and X. Qiu, "Toward accurate and fast irissegmentation for iris biometrics," IEEE Trans. Pattern Anal. Mach.Intell., vol. 31, no. 9, pp. 1670-1684, Sep. 2009.

[7] K. W. Bowyer, K. Hollingsworth, and P. J. Flynn, "Image understandingfor iris biometrics: A survey," Image Vis. Comput., vol. 110, no. 2,pp. 181-307, 2008.

[8] J. Daugman, "New methods in iris recognition," IEEE Trans. Syst., Man,Cybern. B, Cybern., vol. 37, no. 5, pp. 1167-1175, Oct. 2007.

[9] J. R. Matey et al., "Iris on the move: Acquisition of images for irisrecognition in less constrained environments," Proc. IEEE, vol. 94,no. 11, pp. 1936-1947, Nov. 2006.

[10] J. Daugman, "How iris recognition works," IEEE Trans. Circuits Syst.Video Technol., vol. 14, no. 1, pp. 21-30, Jan. 2004.

\section{BIOGRAPHIES}

Ms. Payal D. Mahajan received BE (E\&TC) degree from R.G.P.V Bhopal University. She is now pursuing for ME(VLSI and Embedded System) from Savitribai Phule Pune University, Pune Maharashtra, India.

Asst. Prof. Nandini Dhole is currently working as asst. Prof in RMD Sinhagad School of Engg, Pune. Her main research interests are image processing and digital signal processing. 